\title{
Broadband Frequency ECR Ion Source Concepts with Large Resonant Plasma Volumes
}

\author{
G. D. Alton \\ Oak Ridge National Laboratory ${ }^{*}$ \\ P. O. Box 2008 \\ Oak Ridge, Tennessee 34831-6368
}

\section{Abstract}

New techniques are proposed for enhancing the performances of ECR ion sources. The techniques are based on the use of high-power, variable-frequency, multiplediscrete-frequency, or broadband microwave radiation, derived from standard TWT technology, to effect large resonant "volume" ECR sources. The creation of a large ECR plasma "volume" permits coupling of more power into the plasma, resulting in the heating of a much larger electron population to higher energies, the effect of which is to produce higher charge state distributions and much higher intensities within a particular charge state than possible in present forms of the ECR ion source. If successful, these developments could significantly impact future accelerator designs and accelerator-based, heavy-ion-research programs by providing multiply-charged ion beams with the energies and intensities required for nuclear physics research from existing ECR ion sources. The methods described in this article can be used to retrofit any ECR ion source predicated on B-minimum plasma confinement techniques.

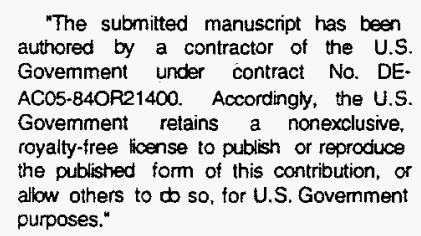

* Managed by Martin Marietta Energy Systems, Inc. under contract DE-AC05-84OR21400 with the U.S. Department of Energy. 


\subsection{Introduction}

Ion sources based on the Electron Cyclotron Resonance (ECR) principle have played major roles in the advancement of accelerator technology since their inception because of their capabilities for generating useful beam intensities of multiply charged ions [1, 2]. Sources of this type offer a number of major advantages over more conventional hot-cathode ion sources, including the following: (1) the source has a long lifetime due to its nonfilamentary (cathode) structure, the component which limits the functional lifetime of the such sources through sputter and chemical erosion processes; (2) the operational stability of the source is unaffected by chemically reactive feed materials; (3) the source operates stably over a wide dynamical pressure range which allows it to be used at low operating pressures for multiply charged ion beam generation ( $\leq 1 \times 10^{-6}$ Torr) commensurate with high-energy nuclear physics research; at higher pressures (> $10^{-5}$ Torr) the source can be used for the generation of high-intensity, low-chargestate ion beams required for many applications. For more details on the state-ofthe-art of ECR ion source technology and their applications, the reader is referred to the proceedings of recent workshops [3-5], ion source conferences $[6,7]$ and the references cited within this article.

Despite progress made in ECR ion source technology over the past several years, the source has not reached its full potential, particularly for multiply charged ion beam generation. We believe that improvements to these sources, in terms of high-charge-state yields and intensities within a particular charge state, all other parameters being equal, can be effected by increasing the physical sizes of the ECR zones in relation to the sizes of the plasma volume. The creation of a large ECR plasma "volume" permits coupling of more power into the plasma, resulting in the heating of a much larger electron population to higher energies and as a consequence, the performance of the ECR ion source may be enhanced in terms of charge state and intensity within a charge state over conventional ECR ion sources. Two methods are readily available for creating large ECR volumes. The 
ECR zones can be increased either by tailoring the magnetic field to achieve a large, uniformly distributed ECR plasma volume $[8,9]$ or by using the broadband microwave radiation techniques described in this article to increase the resonant plasma volumes in sources based on traditional B-minimum confinement magnetic field geometries. The advent of advanced ECR ion sources with charge states and intensities superior to sources presently available would greatly benefit nuclear and atomic physics research, as well as many areas of applied research. Although emphasis is placed on their use for multiply-charged ion beam generation, the principles employed in the new ECR ion source concepts can also be applied for the generation of low-charge-state ion beams.

\subsection{Principles of ECR lon Sources}

The source of energy for plasma generation and maintenance in an ECR ion source is Electron Cyclotron Resonance (ECR) heating of the plasma electrons with microwave radiation. The number density of electrons, the electron energy (temperature), and energy distribution of the electron population are three of the fundamental properties which govern the performance of ECR ion sources in terms of degree of ionization, ion beam intensity, and multiple ionization capabilities. The maximum electron temperature is affected by several processes including the ability of the plasma to adsorb microwave power, the time required to produce heated electrons, the time for thermalization of the "hot" electrons and the ability to contain (confine) the "hot" electrons. The magnetic field geometry and magnetic field strength determine the confinement attributes of the ECR ion source; data accumulated over the years clearly indicate that sources with higher confinement fields generate higher charge state ion beams $[10,11]$.

The ECR zones in any ECR source are limited to regions of the ionization volume where the magnetic field meets the resonance condition, given by 


$$
\omega_{\mathrm{ce}}=\mathrm{Be} / \mathrm{m}=\omega_{\mathrm{rf}}
$$

where $\omega_{\mathrm{ce}}$ is the electron-cyclotron resonant frequency, $\omega_{\mathrm{rf}}$ is the resonant frequency of the microwave power source, $e$ is the electron charge and $m$ the mass of the electron. Whenever the microwave frequency is tuned to the electroncyclotron frequency, electrons can be resonantly excited and thereby given sufficient energy to cause ionization within an evacuated volume. At low collision frequencies (low ambient pressures), some of the electrons are coherently excited and given very high energies which are capable of removing tightly bound electrons and therefore, are responsible for producing multiply charged ions.

For a particular microwave frequency, microwave power can no longer be coupled into the plasma whenever the plasma density reaches a certain value, referred to as the critical density $n_{c}$. The critical density $n_{c}$ occurs whenever the microwave frequency $\omega_{p}$ is identically equal to the plasma frequency $\omega_{r f}$ or $\omega_{r f}=\omega_{p}$. The relation between the critical density $n_{c}$ and the plasma frequency $\omega_{p}$ is given by the following expression:

$$
\mathrm{n}_{\mathrm{c}}=\omega_{\mathrm{p}}^{2} \in_{0} \mathrm{~m} / \mathrm{e}^{2}
$$

Since the ECR condition is met whenever $\omega_{c e}=\omega_{r f}$ (Eq. 1), the critical density increases quadratically with the resonant magnetic field strength $B$. For example, the critical density for $2.45 \mathrm{GHz}$ is $7.45 \times 10^{10} / \mathrm{cm}^{3}$ while the critical density for a 14 $\mathrm{GHz}$ excitation frequency is $2.43 \times 10^{12} / \mathrm{cm}^{3}$. The $2.45 \mathrm{GHz}$ ECR source requires a magnetic field strength of 875 Gauss to meet resonance conditions while the 14$\mathrm{GHz}$ source requires 5000 Gauss fields for resonance to occur. Thus, low frequency sources have lower cutoff densities, and require physically larger plasma chambers for coupling the microwave power but benefit in terms of emittance degradation by the lower magnetic fields in the extraction region of the source while high frequency sources have the decided benefit of higher cutoff densities and small diameter plasma chambers but suffer in terms of emittance 
degradation as a consequence of the strong magnetic fields that exist in the extraction region of the source. (The emittance is degraded in direct proportion to the magnitude of the field strength in the extraction region of the source.)

\subsection{Conventional ECR Ion Sources}

\subsection{General description}

A narrow bandwidth, single frequency source, with the ECR "surface" superposed, is schematically illustrated in Fig. 1 . The source is comprised of a solenoidal magnetic field for confining the plasma in the axial direction, a multicusp magnetic field for confining the plasma in the radial direction, a multimode cavity which serves as the plasma confinement vessel, a single frequency waveguide/microwave injection system for introducing microwave power into the cavity and an ion extraction electrode system for extracting ion beam current from the source. Single-frequency microwave power supplies with frequencies in the range of 2.45 to $14 \mathrm{GHz}$ are typically used to generate and maintain plasmas in these sources; the bandwidths in these power supplies are usually quite narrow (typically, $20 \mathrm{MHz}$ ). The feed material of interest may be introduced in gaseous form or in solid form from ovens or other means (e.g., sputtering or solid rod plasma vaporization). Carrier gases are often used to supplement the discharge in combination with the feed material of interest. The magnetic field distributions are designed to effect a minimum in the $B$ field (B-minimum geometry) for optimum plasma confinement. The usual technique is to provide strong magnetic fields in the axial direction with solenoidal fields located at the ends of the plasma chamber and multicusp (usually sextupole) fields to confine the plasma in the radial direction thus, creating a minimum in the field at the plasma chamber which increases in magnitude from the axis of symmetry toward the walls of the chamber. To illustrate this point, the axial magnetic field distributions of two conventional ECR ion sources are displayed in Figure 2 [12]. As noted, the magnetic field profiles 
continually vary along the axis of symmetry and are approximately parabolic in shape.

\subsection{Limitations in Present ECR Ion Source Technology}

In conventional single frequency ECR ion sources, the shapes, physical sizes, and locations of the ECR zones are determined by the frequency and bandwidth of the microwave radiation and the magnitude and distribution of the magnetic field which meets the ECR condition (Eq. 1). The magnetic field distribution used in traditional ECR ion sources confines the plasma effectively, but severely restricts the physical sizes of the ECR or "hot" zones in relation to the total size of the ionization volume. The ECR zone is usually a thin, fluted, ellipsoidal "surface" which intersects the axis of symmetry of the source at two positions, as illustrated in Fig. 1. Because these ECR "surfaces" are small in relation to the physical size of the ionization chamber, the ECR zones constitute a small fraction of the ionization volume; thus, the absorptivity of microwave radiation by the plasma is determined not by the physical size of the plasma volume but by the size of the ECR zone in the source. Electrons can only be accelerated in the ECR zone; those which leave the ECR zone through scattering have a reduced probability for further stochastic acceleration because of the reduced probability of returning to the zone and therefore, the probability in further acceleration is reduced. Traditional sources with small ECR zones may be more susceptible to these scattering effects which may limit the high energy electron population and thereby limit the ionization rate of the source. The remedy of the problem may be found by increasing the relative size of the ECR zone.

\subsection{Benefits of Large Resonant Volumes}

The principal factors which limit high-charge-state ion production in the ECR source are through charge exchange, wall recombination, ion residence time in the plasma, the bombarding electron current, and the electron temperature. Charge-exchange collisions between ions and neutral atoms reduce the degree of 
ionization and high-charge-state population. High-charge-state particles, ionized in the ECR zones of the source, must necessarily pass through extended regions of unheated plasma before extraction. As a necessary consequence of the requirement of neutrality in the plasma, there is a dynamical charge balance between electron and ion loss processes. Most of the ions recombine at the radial walls of the vacuum chamber and re-enter the plasma as neutrals. The advantage of having a thick ECR zone between the walls of the chamber and the interior of the plasma where the multiply charged ions are extracted may be to improve reionization efficiency of neutrals returning from the walls thereby reducing charge exchange recombination processes within the central plasma region of the source. If the colliding partners are positively ionized, the long range forces and relatively low energies reduces the likelihood of charge transfer in these collisions. The ability to eliminate or drastically reduce the charge exchange process increases the lifetime of a charged particle within a particular charge state, thus, increasing the probability of further ionization. Large ECR volumes result in significantly greater interaction of the microwave radiation with the plasma electrons, both in terms of total power absorptivity and in a more uniform spatial distribution of the absorptivity. The presence of a large ECR zone as well as the additional probability of accelerating larger electron populations to improvements in the higher average energies suggest charge state distributions and ion beam intensities within a particular charge state. This development, if successful, could significantly impact future accelerator designs and accelerator based heavy ion research programs by providing heavy ion beams which presently are too low in intensity for nuclear physics research.

\subsection{Evidence for a Volume Effect}

The "volume" effect has been recently demonstrated incrementally at the Lawrence Berkeley Laboratory by Xie and Lyneis [13]. In this work, the authors simultaneously used $10 \mathrm{GHz}$ and $14 \mathrm{GHz}$ microwave frequencies to excite the plasma in their Advanced ECR (AECR) ion source (Fig. 3) which resulted in moving 
the charge states to higher values by 3 to 4 units for $\mathrm{Bi}$ and $\mathrm{U}$. The data for $\mathrm{U}$ are displayed in Fig. 4. As noted, when operated with two frequencies, the ECR interaction surface areas are increased by $\sim 2$, and as a consequence, the absorptivity of microwave power by the plasma is also increased, making more electrons available for acceleration by the respective RF field. Electrons which scatter out of a particular ECR zone and cross into the second zone can also be further accelerated. The outer $(14 \mathrm{GHz})$ surface may serve to ionize neutrals which result during charged particle recombination at the walls of the chamber and thus, reduce the population of neutrals which would otherwise lower the charge state distribution created in the interior region of the source by the action of the $10 \mathrm{GHz}$ ECR surface. The protective effect of the $14 \mathrm{GHz}$ may offer the best explanation for the enhanced performance of the AECR source because of the unlikely additional stochastic acceleration of electrons that scatter from one zone into another. These experiments provide direct evidence in support of the arguments made in this paper and in Refs. 8 and 9 on the advantages of the "volume" effect. The results of the experiments with the AECR serve to point out the importance of the physical sizes of the ECR zones on the performances of these sources; based on the improvements realized by the presence of only two ECR "surfaces," it appears likely that the charge state distribution and intensity within a particular charge state extracted from sources which have large continuous ECR zones would be significantly improved over those extracted from more conventional sources.. 


\subsection{New ECR Concepts}

The ECR sources described below are based on the use of high-power, variablefrequency, multiple-discrete-frequency, or broad-band microwave radiation, derived from standard traveling wave tube (TWT) technology, to create large ECR "volumes" in conventional ECR source predicated on B-minimum design principles. The ECR "surfaces" in conventional sources are transformed into large ECR "volumes" and are frequency domain complements of the single-frequency, spatial domain ECR source concept, described in Refs. 8 and 9. The size of the ECR "volume" depends on the bandwidth of the variable-frequency or broadband microwave power supply or the number of discrete frequencies introduced into the cavity, as well as the magnetic field distribution with in the plasma volume. By use of fast sweep rate, variable frequency, broadband width or multiple discrete frequency microwave radiation the microwave radiation is adsorbed over a significant region of the plasma volume thereby increasing the probability for acceleration of a greater number of electrons to higher energies.

\subsection{Effecting Broadband Microwave Radiation}

Operation of the traveling wave tube is based on the transfer of energy between an electron beam and an RF wave; the transfer can only be efficient if the electron beam and RF wave are traveling at about the same velocity. Since the microwave travels at a velocity about 100 times that of the electron in free space, a helical structure is used to slow the wave down so that the velocities are about the same. By directing the electron beam along the axis of the helix, the time varying electric field set-up on the helix causes the electron beam energy to vary according to the field strength. The resulting velocity modulation causes the electron beam to form bunches and as the bunches move through the helix, their sizes grow. The helix senses a time varying electric field from the electron bunches which induces an RF wave onto the helix of the same frequency as the initial RF wave, but greatly 
amplified. Power gains up to $70 \mathrm{db}(10,000)$ can be achieved; a single tube can deliver several hundred watts of RF power.

The frequency domain techniques for increasing the resonant volumes in the ECR source concepts described below can be effected with standard traveling wave tube (TWT) technology that has been developed for military applications and more recently developed for processing materials when operated in the microwave regime $[15,16]$. A schematic drawing of a TWT based microwave power supply that could be used to effect increases in the resonant plasma volumes for any of the ECR concepts described in this report is shown in Fig. 5 . The broadband frequency technique of choice can be effected by simply choosing the appropriate complex waveform signal generator.

Advancements have also been made toward the development of broad-band microwave (millimeter wave length) generators in the form of Plasma Wave Tubes (PWTs). These PWTs generate short-pulse, ultra-broad-band sources of RF radiation, applicable for many new uses, including microwave processing of materials $[15,16]$ as well as for the new ECR concepts described in this article. High-power, broadband frequency power supplies been developed for pulsed mode radar jamming applications and have not been used for non-military application to date. However, it is believed that this technology could be adapted to ECR applications. The PWT utilizes the interaction between an electron beam and a time varying plasma to generate kilowatt-power levels $(\sim 10 \mathrm{~kW})$ of radiation at microwave to millimeter-wave frequencies. The electron beam first ionizes the gas to form a plasma and then nonlinearly interacts with the plasma to generate broadband radiation, for example, from 6 to $60 \mathrm{GHz}$. Slew rates of up to $7 \mathrm{GHz} / \mathrm{ms}$ have been measured during a single beam pulse. The radiation has a wide instantaneous bandwidth, typically $10 \mathrm{GHz}$ or wider.

\subsection{The variable frequency ECR ion source concept}


This new ECR ion source concept is based on the use of variable-frequency power to generate and maintain the plasma derived from a TWT based microwave power supply. This source concept is schematically illustrated in Fig. 6. Microwave signals are generated by a voltage controlled oscillator; control voltages are used to vary the oscillator over the complete operating range of the power supply or a selected frequency range within the band width of the power supply; the microwave signal is passed through a preamplifier to provide equal power outputs for a given frequency. The forward power delivered by the traveling wave tube (TWT) and reflected power from the ECR cavity are sampled by the directional couplers and displayed on power meters. The TWT design can deliver power in the $\mathrm{kW}$ range and withstand relatively high levels of reflected power. Since the TWTs are rather expensive, the tolerance to reflected power is very important. Retrofitting existing ECR ion sources can be easily accomplished by replacing the single-frequency microwave power supply with a broad-band, variable-frequency power supply and replacing the wave guide/microwave injection system with a low-loss system which will transmit all frequencies within the band width of the power supply. This type of power supply has been successfully developed by commercial firms and applied for microwave sintering of ceramic materials and other material processing applications $[15,16]$. This design significantly increases the ECR resonant plasma volume resulting in greater absorptivity of the microwave power. All of the concepts, in principle, can be effected by simply modifying the input signal to a traveling wave tube TWT amplifier system.

In the variable-frequency scenario, the complex wave form generator, symbolized in Fig. 5 , is a variable frequency signal generator which is continuously swept through a selected frequency range at a sweep period rate less than $1 \mathrm{~ms}$. The frequency range is chosen to match the bandwidth of the waveguide and the resonance zone frequency distribution of the plasma confinement magnetic field. Since the sweep rate is less than the estimated confinement time $\tau$ of low charge state particles in an ECR ion source, electrons can be accelerated to high energies 
during the sweep cycle. As a consequence, this mode of operation is not expected to significantly compromise the performance of the source relative to that of a source equipped with a constant broad band microwave power supply, provided that the sweep rate is less than the life time of the low charge state components in the plasma. This permits coupling of the total output power into the instantaneously discrete frequency whereas the total output power would be distributed among the frequency components present in multiple-discrete and broadband ECR ion source concepts such as described below. By sweeping the frequency at a sufficiently fast rate, power can be continually and more uniformly distributed over the resonant plasma volume. The ability to homogeneously distribute the power over the resonant plasma volume suggests that the stability of operation would be improved over present forms of the source. Saturation effects are avoided since the ECR zone continually moves due to the changing frequency. However, it is not yet known whether or not this method will produce a stable discharge. It may be desirable to use two or more variable frequencies phased so that one signal is increasing while the other is decreasing so as to create a more homogeneous plasma.

\subsection{Broadband frequency ECR ion source concept}

This scenario is also illustrated in the source concept shown in Fig. 6. This scheme, again, would incorporate TWT technology with an appropriately chosen broadband signal generator as the complex waveform generator in Fig 5 . For this scenario, the power would be distributed across the band width of the frequency spectrum. The bandwidth would again be chosen to match the wave guide bandwidth and resonant frequency distribution of the magnetic field associated with the particular ECR ion source.

\subsection{Multiple, discrete-frequency ECR ion source concept}


The ECR plasma volume also can be increased by exciting the plasma with multiple, discrete frequencies as illustrated in Fig. 7. For this scenario, the complex wave form generator is a multiple, discrete frequency generator selected to fill the bandwidth of the wave guide. Again the set of frequencies is chosen to fit into the resonance frequency distribution of the particular ECR ion source. This technique can be easily effected by use of traveling wave tube (TWT) technology. The total power output would be distributed among the frequencies which are used. Two klystron based microwave power supplies could also be utilized, much like the system utilized at LBL [13], to achieve a larger ECR zone within the source. However, this course of action would appear to be impractical for most situations because of the expense of microwave power supplies, wave guides and auxiliary equipment and the resulting complexity of operation of the source. The expense can be reduced and the complexity of operation eliminated by use of a single, high power TWT based amplification system equipped with a multiple discrete frequency generator that fills the band width of a broadband wave guide used for injection into the source. Of course, these wave forms could be phased randomly or in some programmable way if beneficial to operational stability.

\subsection{Discussion}

Perhaps the most practical aspect of the broadband frequency techniques described in this article is that they can be utilized to relatively inexpensively transform present "surface" ECR ion sources into "volume" sources. By use of these techniques, traditional single frequency sources can be converted from resonant "surface" sources to resonant "volume" sources, thus, permitting the coupling of more power into the plasma resulting in the heating of a much larger electron population to higher energies than presently possible in conventional ECR ion sources. The ability to quickly ionize a large fraction of the neutral population that results from recombination of the multiply charged ions which strike the walls of the vacuum chamber may significantly reduce the rate of charge exchange, thereby 
increasing the residence time of an ion in a given charge state, which increases the probability for subsequent and further ionization. By increasing the size of the ECR zone on the low frequency side of the spectrum, the resonant plasma volume will be closer to the axis of extraction where the ion beam can be extracted more efficiently resulting in higher beam intensity. Based on experimental evidence provided in Ref. 13 and the results of computation studies described in Refs. 8 and 9 , it appears likely that the performances of ECR ion sources can be enhanced by increasing the relative sizes of the resonant plasma volumes in these sources. 


\section{References:}

1. R. Geller, B. Jacquot, and C. Jacquot, Plasma Phys. Controlled Nucl. Fusion Res. (1972), Proc. Int. Conf., 4th, Madison, Wis. CN-28, G6 (1971).

2. R. Geller, Trans. Nucl. Sci. NS-23, 904 (1976).

3. Proceedings of the Tenth International Workshop on ECR Ion Sources (Knoxville, TN, Nov. 1-2, 1990), edited by F. W. Meyer and M. I. Kirkpatrick, CONF-9011136, 1991.

4. Proceedings of the Eleventh International Workshop on ECR Ion Sources (Groningen, The Netherlands, May 6-7, 1993), edited by A. G. Drentje, KVI Report 996 (1993).

5. Proceedings of the Twelfth International Workshop on ECR Ion Sources, edited by M. Sekiguchi an T. Nakagawa, (The Institute of Physical and Chemical Research (RIKEN), April 25-27,1995, Wakoshi, Japan), INS-J-182 (1995).

6. Proceedings of the Fourth International Conference on Ion Sources, edited by B. H. Wolf, (30 September-4 October, 1991, Bensheim, Germany) Rev. Sci. Instrum. 63 (1995).

7. Proceedings of the Fifth International Conference on Ion Sources, edited by C. Chen and W. Zhao, (31 August-4 September 1993, Beijing, China) Rev. Sci Instrum. 65 (1994).

8. G. D. Alton and D. N. Smithe, Rev. Sci. Instrum. 65 (1994) 775.

9. G. D. Alton and D. N. Smithe, in Proceedings of the Twelfth International Workshop on ECR lon Sources, edited by M. Sekiguchi and T. Nakagawa, (The Institute of Physical and Chemical Research (RIKEN), April 25-27, 1995, Wakoshi, Japan), INS-J-182 (1995) 100.

10. D. Hitz, in Proceedings of the Twelfth International Workshop on ECR Ion Sources, edited by M. Sekiguchi an T. Nakagawa, (The Institute of Physical and Chemical Research (RIKEN), April 25-27,1995, Wakoshi, Japan), INS-J182 (1995) 161

11. G. Ciavola, S. Gammino, T. Antaya and K. Harrison, in Proceedings of the Twelfth International Workshop on ECR Ion Sources, edited by M. Sekiguchi an T. Nakagawa, (The Institute of Physical and Chemical Research (RIKEN), April 25-27, 1995, Wakoshi, Japan), INS-J-182 (1995) 156. 
12. P. Bricault, P. Sortais, M. Bisch, P. Leherissier, R. Lecroy, J. Y. Pacquet, and J. P. Rataud, Rev. Sci. Instrum. 63 (1992).

13. Z. Q. Xie and C. M. Lyneis (to be published in Rev. Sci. Instrum.)

14. Y. Jongen and C. M. Lyneis, in The Physics and Technology of lon Sources, edited by I. G. Brown (Wiley, New York, 1989).

15. "2 to $18 \mathrm{GHz}$ Broad Band Microwave Heating Systems," R. J. Lauf, D. W. Bible, A. C. Johnson, and C. A. Eberleigh, Technical Features, Microwave Journal, November 1993.

16. U.S. Patent No. 5, 321, 222, "Variable Frequency Microwave Furnace Systems," D. W. Bible and R. J. Lauf.

17. J. Santoru, J. M. Butler, D. M. Goebel, and R. W. Schumacher, IEEE Trans. on Plasma Sci. 22 (1994) 593.

\section{DISCLAIMER}

This report was prepared as an account of work sponsored by an agency of the United States Government. Neither the United States Government nor any agency thereof, nor any of their employees, makes any warranty, express or implied, or assumes any legal liability or responsibility for the accuracy, completeness, or usefulness of any information, apparatus, product, or process disclosed, or represents that its use would not infringe privately owned rights. Reference herein to any specific commercial product, process, or service by trade name, trademark, manufacturer, or otherwise does not necessarily constitute or imply its endorsement, recommendation, or favoring by the United States Government or any agency thereof. The views and opinions of authors expressed herein do not necessarily state or reflect those of the United States Government or any agency thereof. 


\section{Figure Captions}

Fig. 1. ORNL-DWG 95M-10050. Schematic drawing of a single- frequency, Bminimum geometry ECR ion source for multiply-charged ion beam generation.

Fig. 2. ORNL-DWG 94Z-8364B. Axial magnetic field profiles for the ECR3/ CAPRICE and NEOMAFIOS ECR sources described in Ref. 12.

Fig. 3. ORNL-DWG 95M-6661. Schematic illustration of the Berkeley AECR ion source [14] operated with two klystron based microwave power supplies with output frequencies of $10 \mathrm{GHz}$ and $14 \mathrm{GHz}$, respectively [13].

Fig. 4. ORNL-DWG 95M-6162. Charge state distributions for $U$ from the Berkeley AECR ion source when operated with single frequency (14 GHz; curve 1) and double frequency (14 GHz +10 GHz, curve 2) (Ref. 13 ).

Fig 5. ORNL-DWG 95M-6163. Schematic diagram of a broadband, TWT based power, microwave power supply system for creating large resonant plasma zones in ECR ion sources with B-minimum magnetic field configurations. The complex wave form generator can be a fast-sweep-rate, variable-frequency microwave generator; a broadband frequency (noise) generator or; a multiple, discrete frequency microwave generator.

Fig. 6. ORNL-DWG 95M-6188. Schematic illustration of an ECR ion source with a large, uniformly-distributed, ellipsoidal resonant plasma volume. The microwave power supply is assumed to be a broadband TWT based system driven with either of the following complex wave form generators: a) a variable frequency at fast sweep rate ( $<1 \mathrm{~ms})$ or; b) a broadband noise generator.

Fig. 7 ORNL-DWG 95M-10049. Schematic illustration of an ECR ion source with multiple, ellipsoidal resonant surfaces. The microwave power supply is assumed to be a broadband TWT based system driven with multiple discrete frequencies. 


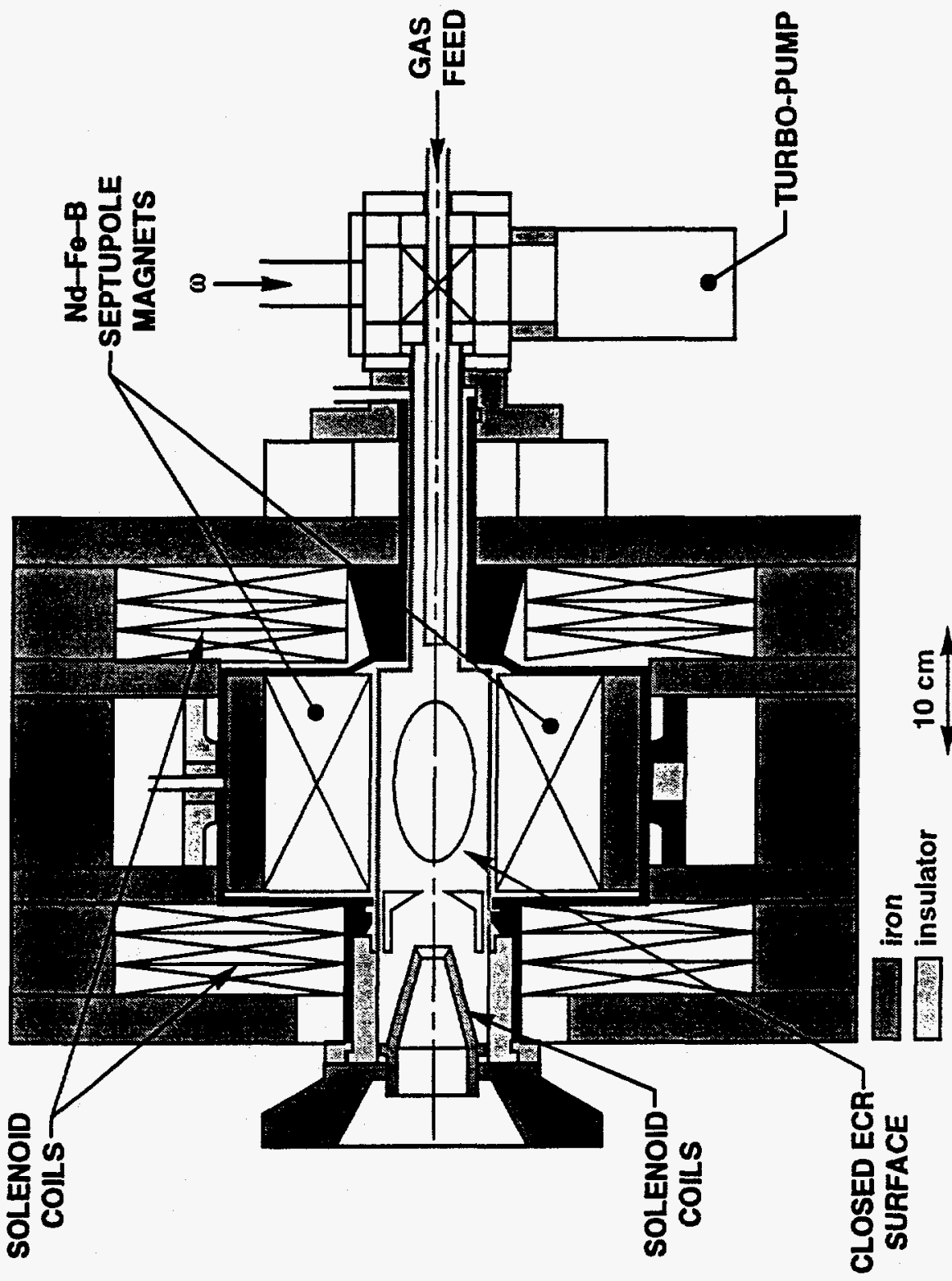




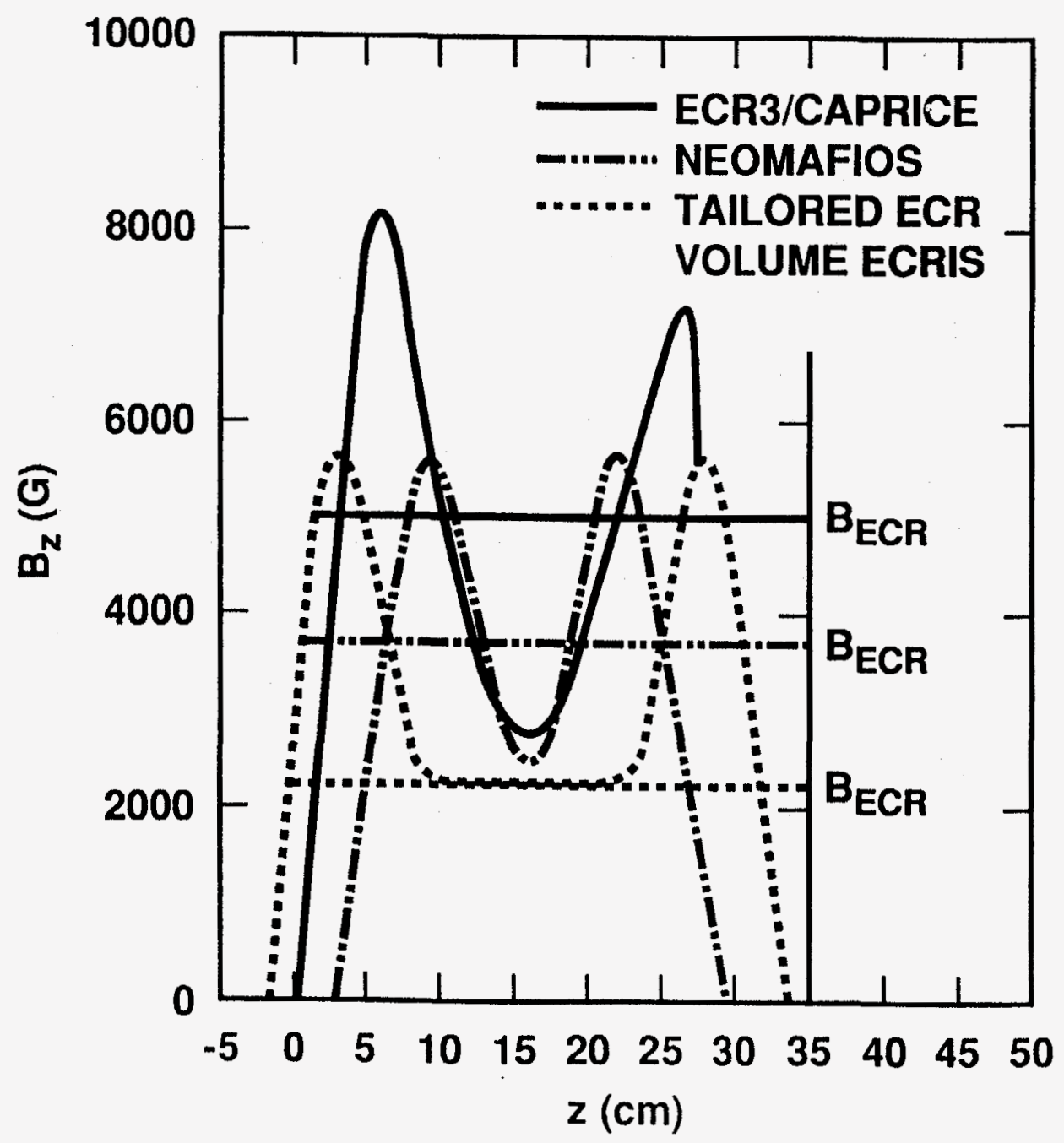

Fig. 2 
SCHEMATIC DRAWING OF THE BERKELEY ECR ION SOURCE OPERATES WITH TWO FREQUENCIES $(10 \mathrm{GHz}$ and $14 \mathrm{GHz})$ MICROWAVE POWER

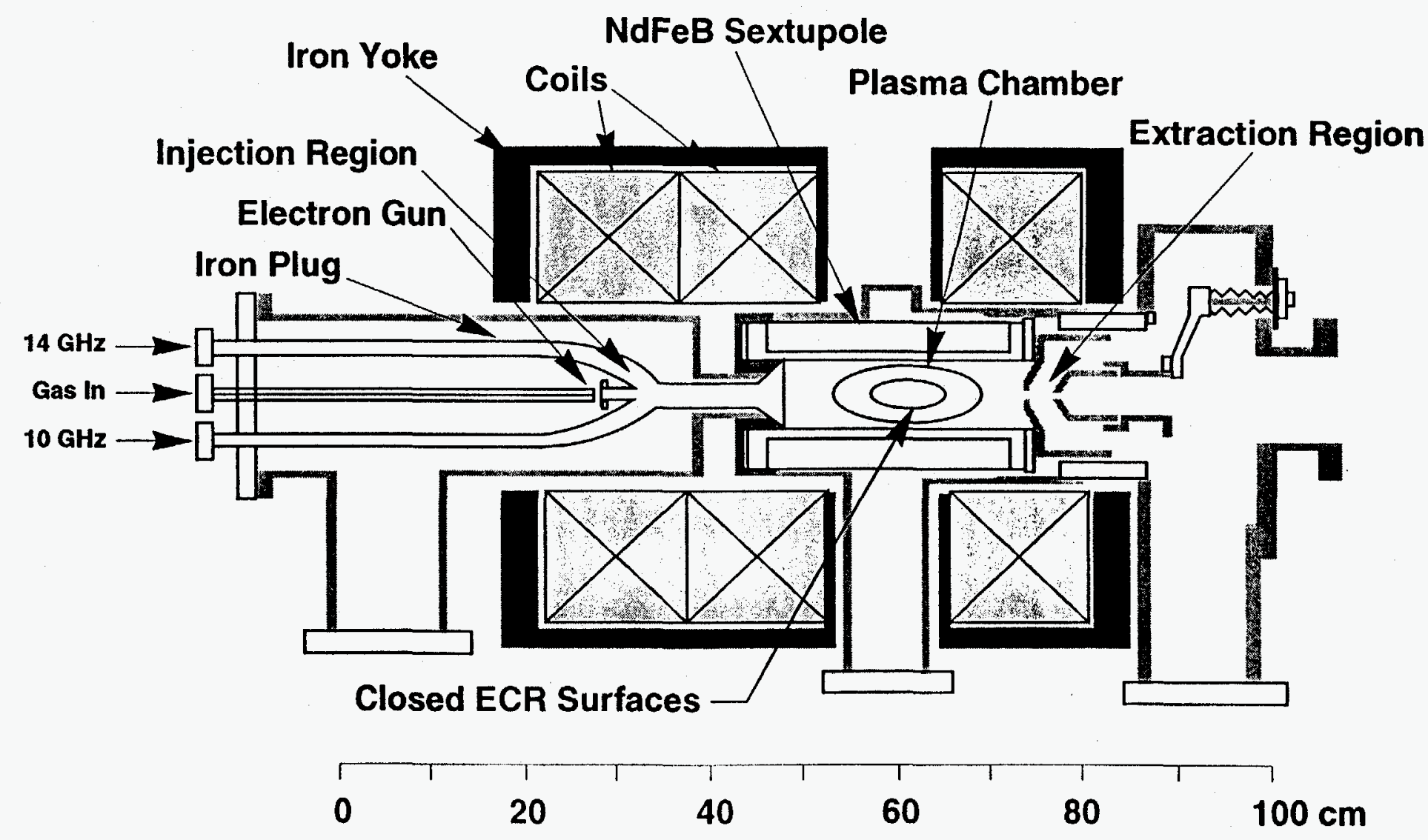




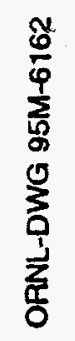

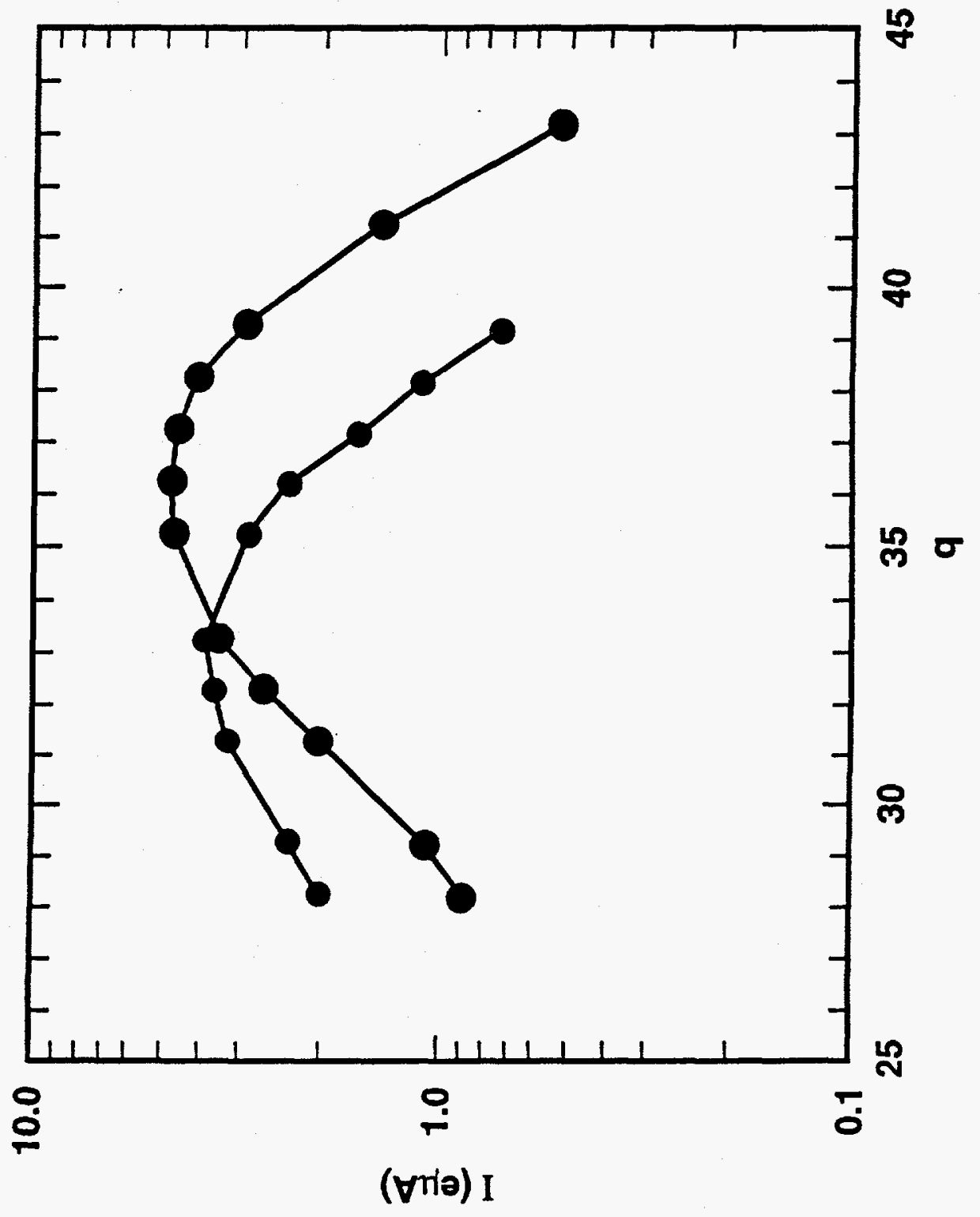




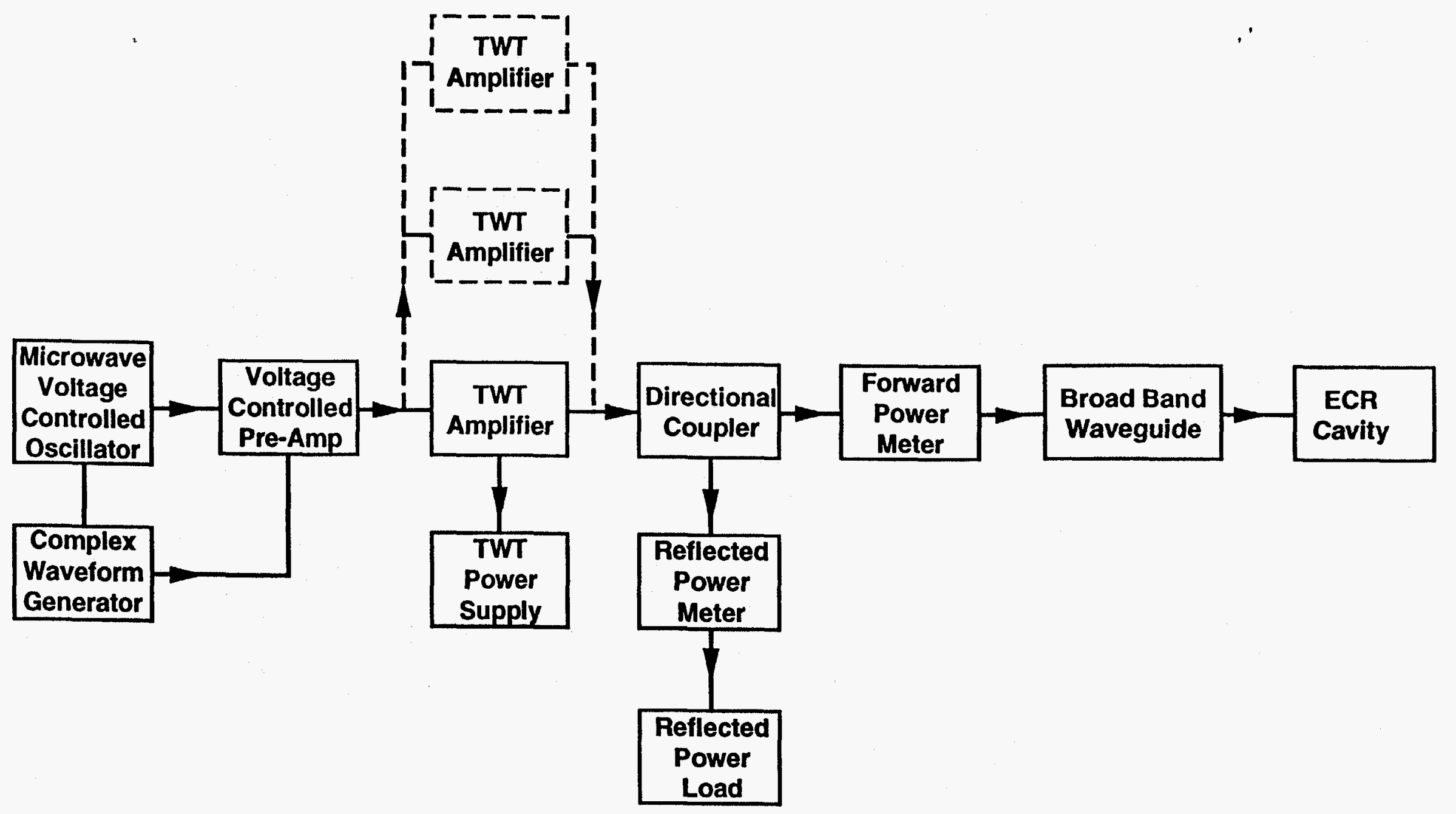

Fig. 5 

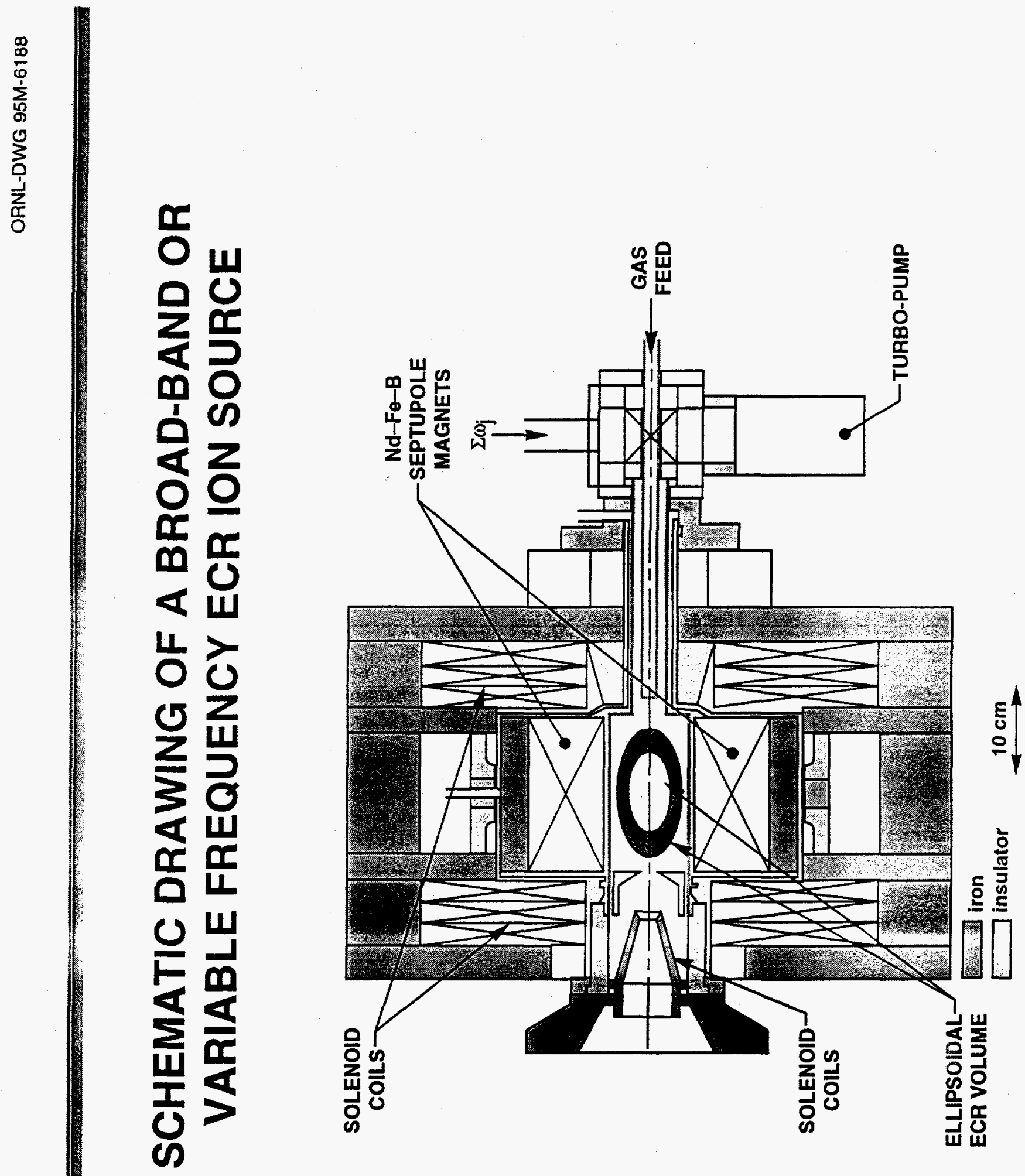

这 


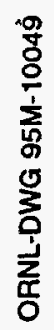

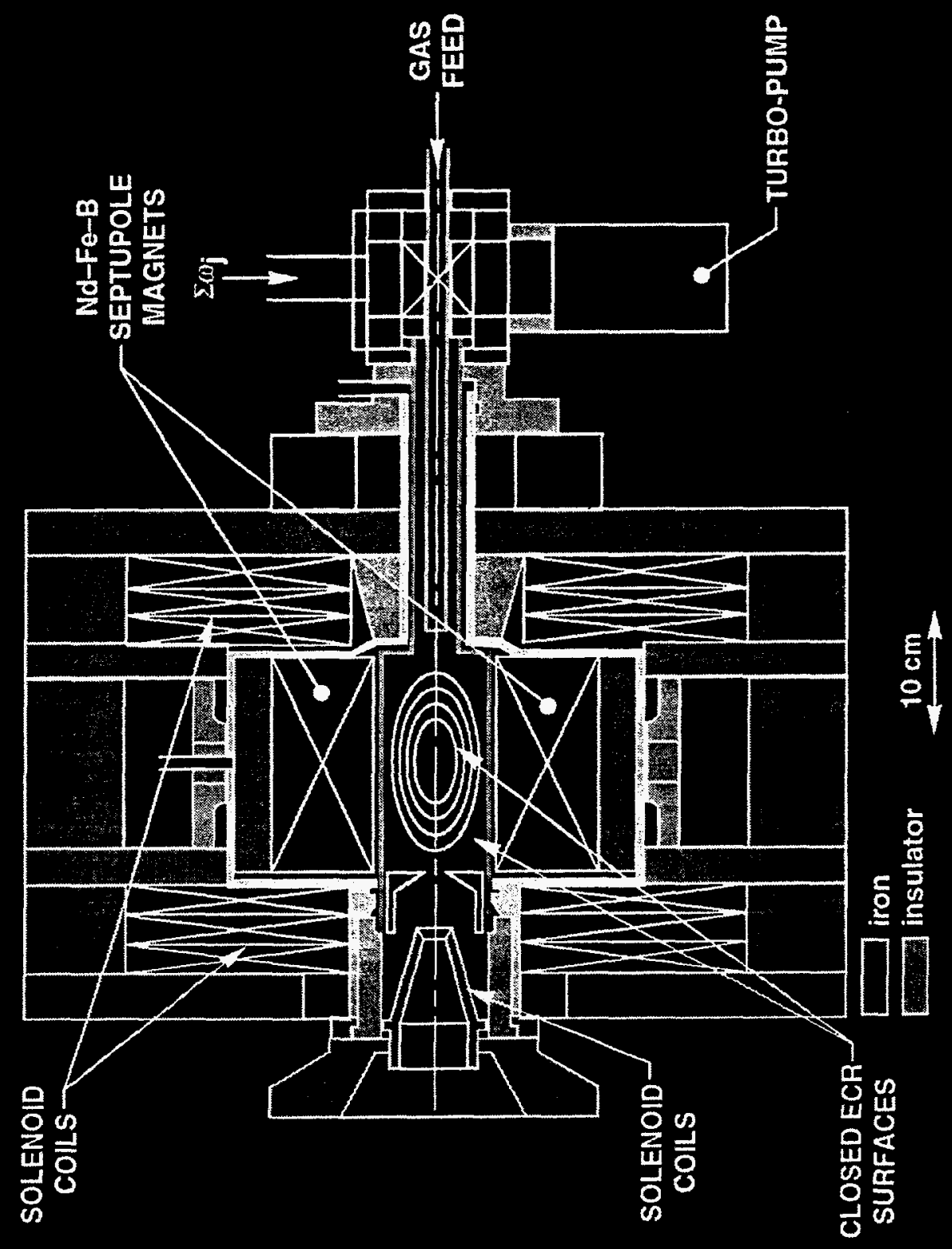

